\title{
From basic medicine to clinical application in exercise rehabilitation
}

\author{
Chang-Ju Kim (iD https://orcid.org/0000-0003-4749-5795
}

"From basic medicine to clinical application in exercise rehabilitation."

This is the theme of the Autumn Conference of the Korean Society of Exercise Rehabilitation to be held on September 16, 2017 at Kyung Hee University.

Rehabilitation therapy in the broad sense refers to any treatment that achieves and maintains the optimal physical, sensory, intelligent, psychological, and social levels for a person with a disability. It also means all the therapies to restore the patient's quality of life when the patient is suffering from pain, temporary illness, or trauma without any disability.

On the other hand, the narrow meaning of rehabilitation treatment can be considered distinguishably to the treatment-oriented medicine. Rehabilitation therapy refers to the treatment of patients' activities by mediating and using physical stimuli to restore and maintain physical function, whereas treatment-oriented medicine mainly uses drugs or surgery.

Exercise rehabilitation is a part of rehabilitation therapy. Current exercise therapy is limited to the act of helping the therapist to passively move the patient's joints, or to educate and assist in walking and help the patient's exercise function to be restored and maintained. As is well known, the effects of exercise on the human are very broad, but the diseases that can actually apply exercise rehabilitation are still very limited.

It is also important to verify the effects of exercise rehabilitation on various diseases in order to broaden the therapeutic scope of exercise rehabilitation. In addition, supplementary effects on surgery and medication are also an important function of exercise rehabilitation. These kinds of papers are contributing to the Journal of Exercise Rebabilitation (JER) all over the world.

In this issue of JER, a lot of papers on the clinical efficacy of exercise rehabilitation, including obesity, pulmonary function, pain, and muscle damage, have been reported from diverse countries. Of course, articles on the basic medical science such as neurogenesis, brain-derived neurotrophic factor, and ketone body are also included in this issue.

Nowadays, basic medical research for the evaluation of the mechanisms of exercise rehabilitation is a necessary task for the development of a new therapeutic area of exercise rehabilitation. The basic medical study which verified the effect of exercise after stem cell treatment or the change of brain after forcible exercise with the help of a mechanical device suggested the possibility of applying it to clinical practice of exercise rehabilitation in the future. JER welcomes bold and active basic science papers for the identification of the mechanisms of rehabilitation exercise as above.

Korean Society of Exercise Rehabilitation aims not only academic progress of exercise rehabilitation through JER but also challenging development of exercise rehabilitation through academic conference. In this Autumn Conference of the Korean Society of Exercise Rehabilitation, we prepared the contents of the link between basic medicine and clinical application of exercise rehabilitation. Through this conference, we hope that the development of small but meaningful integration of basic medicine to clinical application will be achieved. 


\section{CONFLICT OF INTEREST}

No potential conflict of interest relevant to this article was reported.
President of the Korean Society of Exercise Rehabilitation Department of Physiology, College of Medicine Kyung Hee University 26 Kyungheedae-ro, Dongdaemun-gu, Seoul 02447, Korea E-mail: changju@khu.ac.kr 